\title{
Comparative Studies of Histochemical Usefullness of Tetranitro-BT (TNBT) and nitro-BT (NBT)
}

\author{
Kazuo Ogawa and Yasuyuki Shinonaga \\ Department of Anatomy, Kyoto University School of Medicine.
}

In 1962 Tsou et al reported the usefulness of a new tetrazolium salt, Tetranitro-BT $\left(2,2,,^{\prime}\right.$ 5,5'-tetra-p-nitrophenyl -3,3'- (3,3'-dimethoxy-4,4'-biphenylene)ditetrazolium chlovide] as a good reagent for histochemistry of oxidative enzymes.

The authors have demonstrated succinic dehydrogenase system and DPNH diaphorase activity in kidney, cerebellum and Hela cells using TNBT and NBT.

As a result, TNBT was better than NBT in following two items.

(1) Size of formazan granules of TNBT was more homogeneous and finer than those of NBT.

(2) Solubility of formazan of TNBT was lower than that of NBT.

\section{Studies of New Buffer Solution in Histochemistry}

\author{
Hideo Takamatsu, Akira Kitamura, Masakazu Takesawa \\ Takashi IKedA and Toshio Tominaga
}

The Department of Pathology, Tuberculosis Research Institute, Kyoto University.

(Director : Prof. H. Takamatsu)

In experiments of enzyme reactions, buffer solutions are usually added to the test solution for keeping the hydrogen ion concentration in a certain range. Each constituent of various buffers has its own properties as a chemical

Ac. : $1 / 10-\mathrm{N}$ Acetic Acid

Table 1.

NTA. : 2Na. 1/10-Mol. Nitrilotriacetic Acid Disodium Salt.

\begin{tabular}{|c|c|c|}
\hline Ac & : NTA. $2 \mathrm{Na}$ & $\mathrm{pH}$ \\
\hline 7 & $:$ & 3.5 \\
\hline 6 & $: \quad 4$ & 3.6 \\
\hline 5 & : 5 & 3.7 \\
\hline 4 & $: \quad 6$ & 3.8 \\
\hline 3 & $: \quad 7$ & 3.9 \\
\hline 2 & : 8 & 4.0 \\
\hline 1 & $: \quad 9$ & 4.3 \\
\hline 0 & : 10 & 7.8 \\
\hline
\end{tabular}

